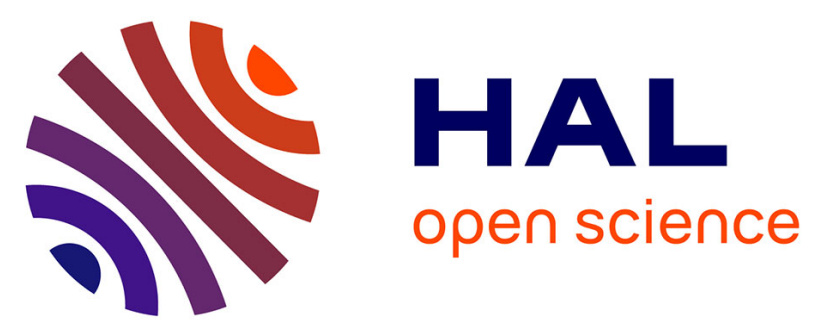

\title{
Spatial Laplace transform for complex wavenumber recovery and its application to the analysis of attenuation in acoustic systems
}

A. Geslain, Samuel Raetz, M. Hiraiwa, M. Abi Ghanem, P. Wallen, A. Khanolkar, N. Boechler, Jérôme Laurent, C. Prada, Aroune Duclos, et al.

\section{To cite this version:}

A. Geslain, Samuel Raetz, M. Hiraiwa, M. Abi Ghanem, P. Wallen, et al.. Spatial Laplace transform for complex wavenumber recovery and its application to the analysis of attenuation in acoustic systems. Journal of Applied Physics, 2016, 120 (13), 10.1063/1.4963827 . hal-01445395

\section{HAL Id: hal-01445395 \\ https://u-bourgogne.hal.science/hal-01445395}

Submitted on 20 Feb 2018

HAL is a multi-disciplinary open access archive for the deposit and dissemination of scientific research documents, whether they are published or not. The documents may come from teaching and research institutions in France or abroad, or from public or private research centers.
L'archive ouverte pluridisciplinaire HAL, est destinée au dépôt et à la diffusion de documents scientifiques de niveau recherche, publiés ou non, émanant des établissements d'enseignement et de recherche français ou étrangers, des laboratoires publics ou privés. 


\title{
Spatial Laplace transform for complex wavenumber recovery and its application to the analysis of attenuation in acoustic systems
}

\author{
A. Geslain, ${ }^{1}$ S. Raetz, ${ }^{2}$ M. Hiraiwa, ${ }^{3}$ M. Abi Ghanem, ${ }^{3}$ S. P. Wallen, ${ }^{3}$ A. Khanolkar, ${ }^{3}$ \\ N. Boechler, ${ }^{3}$ J. Laurent, ${ }^{4}$ C. Prada, ${ }^{4}$ A. Duclos, ${ }^{2}$ P. Leclaire, ${ }^{1}$ and J.-P. Groby ${ }^{2, a)}$ \\ ${ }^{1}$ DRIVE EA1859, Univ. Bourgogne Franche Comté, 58000 Nevers, France \\ ${ }^{2}$ Laboratoire d'Acoustique de l'Université du Maine, UMR-6613 CNRS/Université du Maine, Avenue Olivier \\ Messiaen, 72085 Le Mans, France \\ ${ }^{3}$ Department of Mechanical Engineering, University of Washington, Seattle, Washington 98195, USA \\ ${ }^{4}$ Institut Langevin, CNRS, ESPCI Paris, PSL Research University, 1 rue Jussieu, 75005 Paris, France
}

\begin{abstract}
We present a method for the recovery of complex wavenumber information via spatial Laplace transforms of spatiotemporal wave propagation measurements. The method aids in the analysis of acoustic attenuation phenomena and is applied in three different scenarios: (i) Lamb-like modes in air-saturated porous materials in the low $\mathrm{kHz}$ regime, where the method enables the recovery of vis-coelastic parameters; (ii) Lamb modes in a Duralumin plate in the MHz regime, where the method demonstrates the effect of leakage on the splitting of the forward $S_{1}$ and backward $S_{2}$ modes around the ZeroGroup Velocity point; and (iii) surface acoustic waves in a two-dimensional microscale granular crystal adhered to a substrate near $100 \mathrm{MHz}$, where the method reveals the complex wave-numbers for an out-of-plane translational and two inplane translational-rotational resonances. This method provides physical insight into each system and serves as a unique tool for analyzing spatio-temporal measurements of propagating waves.
\end{abstract}

\section{INTRODUCTION}

Understanding the dispersive and dissipative properties of materials is critical to the study of wave phenomena. Extracting complex wavenumber information is important, particularly in the context of understanding wave attenuation. In addition to dispersive effects, such as the existence of band gaps, wave attenuation can be caused by factors such as geometric attenuation or intrinsic material loss (e.g., heat dissipation). In any of these cases, the wave attenuation can be interpreted in terms of complex wavenumbers. The recovery of complex wavenumbers is of particular interest for the characterization of the viscoelastic properties of materials, in systems such as thin-films, ${ }^{1,2}$ or coated plates, ${ }^{3}$ the study of mode interactions, i.e., hybridization or repulsion, ${ }^{4-6}$ or the recovery of complex band structures arising from structural periodicity or resonant elements. ${ }^{4,7}$ The dispersion of waves propagating through materials is typically interpreted in the context of frequency and wavenumber domain information and obtained from discrete spatiotemporal data via discrete Fourier transforms and related methods. ${ }^{9-14}$ However, such techniques typically only supply real wavenumber information (or their magnitudes) from twodimensional, discrete, spatiotemporal wave propagation information, such as may be obtained from scanned receiver measurements. Several methods have been proposed to characterize wave attenuation and extract complex wavenumber information; ${ }^{15-22}$ however, each has restrictions, as: (i) they are usually based on measurements of wave amplitude decrease

\footnotetext{
${ }^{\text {a) }}$ Author to whom correspondence should be addressed. Electronic mail: jean-philippe.groby@univ-lemans.fr
}

with respect to time, ${ }^{15-18}$ (ii) they are iterative methods applied in space, like the modified Prony method, ${ }^{19}$ (iii) the number of modes has to be known in advance or a unique mode has to be isolated, ${ }^{15-17,20,21,23}$ (iv) the modes contributing significantly to the signal are presumed to not interact or overlap with one another, or (v) they must include a third dimension of information, such as would be the case in an experiment with a scanned emitter and a receiver. ${ }^{21,22}$

In this work, a method presenting none of these restrictions is proposed and applied to the analysis of complex attenuation phenomena in the scanned spatiotemporal measurements of three acoustic systems. After presenting the implementation of the method making use of a spatial Laplace transform in Sec. II, we discuss its application in the following three diverse scenarios. In Sec. III, the method is applied to the study of low frequency $(200 \mathrm{~Hz}-4095 \mathrm{~Hz})$ guided elastic waves in porous materials, which are highly dissipative systems such that the wavenumbers associated with each mode are complex. The method is utilized to characterize skeleton (matrix) viscoelastic parameters. In Sec. $\mathrm{IV}$, the method is applied to the analysis of Zero-Group Velocity (ZGV) Lamb modes at $\mathrm{MHz}$ frequencies (1.85 $\mathrm{MHz}-2 \mathrm{MHz}$ ) in a Duralumin plate. The ZGV modes are composed of two interfering counter-propagating Lamb modes, and the method reveals mode separation due to leakage and their associated complex wavenumbers, along with motivating the need for more complex multilayer models. Finally, in Sec. V, the method is applied to characterize the resonant attenuation of high frequency $(10 \mathrm{MHz}-400 \mathrm{MHz})$ surface acoustic waves (SAWs) propagating through a two-dimensional (2D) microscale granular crystal adhered to a substrate with three contact-based resonances. While 
hybridization due to the out-of-plane mode can be seen using a usual spatial Fourier transform, ${ }^{24}$ the two combined rotational and in-plane translational resonances are only noticeable by studying the attenuation of the modes, which is further highlighted by this method.

\section{THE RECOVERY OF COMPLEX WAVENUMBERS: THE SLaTCOW METHOD}

The acronym of the proposed method is SLaTCoW for Spatial LAplace Transform for COmplex Wavenumber recovery. In what follows, we focus on the extraction of complex wavenumber information from guided elastic wave measurements. However, we note that the SLaTCoW method is sufficiently general that it may be applied to wave fields of any type. Assume that a wave field $\xi$ has been recorded discretely along a line of length $L$ and has a time dependence of $e^{-\mathrm{i} \omega t}$, where $\omega=2 \pi f$ is the angular frequency. This field (after neglecting the branch integrals arising from the application of the residue theorem) can be written in the frequency domain as the sum of the contributions of each of the modes ( $\omega$ is dropped for clarity)

$$
\xi(x)=\sum_{m \in \mathcal{M}} \tilde{\xi}^{m} \exp \left(\mathrm{i} K^{m} x\right) \Pi(x, L),
$$

where $x$ is the spatial coordinate, $\tilde{\xi}^{m}$ is the complex amplitude of the $m$-th mode, $K^{m}$ is the complex wavenumber of the $m$-th mode, $\mathcal{M}$ is the set of modes, and $\Pi(x, L)$ is the gate function equal to 1 when $x \in[0, L]$ and equal to 0 elsewhere. The complex wavenumber is defined as $K^{m}=k_{r}^{m}$ $+\mathrm{i} k_{i}^{m}$, with $k_{r}^{m}>0$ and $k_{i}^{m}>0$, such that Eq. (1) only involves forward propagating modes. Applying the usual spatial Fourier transform only enables the recovery of $k_{r}^{m}$. In order to recover both real and imaginary parts of $K^{m}$, a spatial Laplace transform is applied to $\xi(x)$ denoted $\Xi(s)$ $=\int_{-\infty}^{\infty} \xi(x) \exp (-s x) d x$, where $s$ is a complex number wavenumber parameter $s=s_{i}+\mathrm{i} s_{r}$, with real numbers $s_{i}$ and $s_{r}$. This spatial Laplace transform takes the form

$$
\begin{aligned}
\Xi(s) & =\sum_{m \in \mathcal{M}} \tilde{\xi}^{m} \int_{0}^{L} \exp \left[\left(\mathrm{i} K^{m}-s\right) x\right] d x \\
& =L \sum_{m \in \mathcal{M}} \tilde{\xi}^{m} \exp \left[\left(\mathrm{i} K^{m}-s\right) L / 2\right] \frac{\sinh \left[\left(\mathrm{i} K^{m}-s\right) L / 2\right]}{\left(\mathrm{i} K^{m}-s\right) L / 2} .
\end{aligned}
$$

The meaning of the spatial Laplace transform of the $m$-th mode is ensured only if $s_{i} \geq-k_{i}^{m}$ because this ensures the energy decay of each mode. Thus, the upper half space, the lower bound of which is the maximum value of $-k_{i}^{m}$ with $m \in \mathcal{M}$, is the only admissible half space in the complex $s$-plane and defines the region of absolute convergence of the Laplace transform. We note that the slice of $\Xi(s)$ in the complex $s$ plane at $s_{i}=0$ exactly corresponds to the spatial Fourier transform $\operatorname{TF}\left(s_{r}\right)$ of a finite window. Along this line, Eq. (2) reduces to $\operatorname{TF}\left(s_{r}\right)=L \sum_{m \in \mathcal{M}} \tilde{\xi}^{m} \exp \left[\mathrm{i}\left(k_{r}^{m}-s_{r}+\mathrm{i} k_{i}^{m}\right) L / 2\right]$ $\operatorname{sinc}\left[\left(k_{r}^{m}-s_{r}+\mathrm{i} k_{i}^{m}\right) L / 2\right]$, where $\operatorname{sinc}(x)=\sin (x) / x$, as can be seen in Fig. 1.

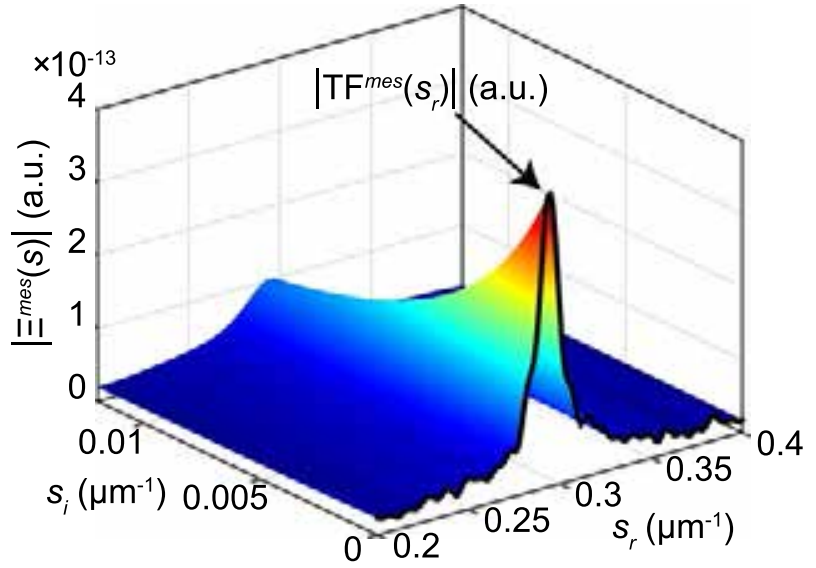

FIG. 1. Laplace transform $\Xi^{\text {mes }}(s)$ corresponding to $f=149 \mathrm{MHz}$, for the scanned measurements discussed in Section V. The solid black line shows the corresponding amplitude of the spatial Fourier transform $\operatorname{TF}^{m e s}\left(s_{r}\right)$.

The problem when trying to recover $K^{m}$ is twofold: the amplitude and phase of the mode are unknown and the position of $-k_{i}^{m}$ in the complex $s$-plane is by definition unknown. Therefore, we will focus the analysis on the upper half space $s_{i} \geq 0$ where no mode $K^{m}$ is included, thus making the method stable by preventing the divergence of the transform due to the poles. Inspired by previous works on the recovery of the reflection coefficients of higher order modes propagating in a square cross-section impedance tube, ${ }^{25}$ the recovery of $K^{m}$ is performed for each frequency by minimizing the following cost function:

$$
\begin{aligned}
& F\left(\left|\tilde{\xi}^{m}\right|, \phi^{m}, k_{r}^{m}, k_{i}^{m}, \mathcal{M}\right) \\
& =\sum_{s_{r}} \sum_{s_{i}}\left|\Xi^{m e s}(s)-L \sum_{m \in \mathcal{M}}\right| \tilde{\xi}^{m} \mid \\
& \quad \times \exp \left(\mathrm{i} \phi^{m}\right) \times \exp \left(\left(\mathrm{i}\left(k_{r}^{m}+\mathrm{i} k_{i}^{m}\right)-s_{i}-\mathrm{i} s_{r}\right) L / 2\right) \\
& \quad \times \frac{\sinh \left(\left(\mathrm{i}\left(k_{r}^{m}+\mathrm{i} k_{i}^{m}\right)-s_{i}-\mathrm{i} s_{r}\right) L / 2\right)}{\left(\mathrm{i}\left(k_{r}^{m}+\mathrm{i} k_{i}^{m}\right)-s_{i}-\mathrm{i} s_{r}\right) L / 2} \mid,
\end{aligned}
$$

where $\Xi^{\text {mes }}(s)$ is the spatial Laplace transform of the measured field $\xi(x)$, and $\left|\tilde{\xi}^{m}\right|$ and $\phi^{m}$ are, respectively, the theoretical amplitude and phase of the $m$-th mode. Note that the $\mathcal{L}_{1^{-}}$ norm is used in Eq. (3) because it leads to quite similar results as the usual $\mathcal{L}_{2}$-norm. The latter may be preferable when analyzing signals with a low signal-to-noise ratio. The minimization is performed under constraints with the Nelder-Mead simplex algorithm $\left({ }^{\circledR}\right.$ Matlab function fminsearchbnd). In Eq. (3), it can be seen that there are $4|\mathcal{M}|$ number of unknowns, corresponding to the wave amplitude, phase, and real and imaginary parts of the complex wavenumber $K^{m}$ of each mode. To solve for these unknowns, we first determine the number of modes to be recovered, along with their real wavenumbers, amplitudes, and phases, by looking at the spatial Fourier transform for each frequency. As an example, the solid black line in Figure 1 shows the amplitude of the spatial Fourier transform $\mathrm{TF}\left(s_{r}\right)$, corresponding to $f=149 \mathrm{MHz}$, for the scanned measurements discussed in Section V. It can be seen from the Fourier spectrum that there is one clear 
peak (mode) at this frequency, with real wavenumber $k_{r}^{1}$ $\approx 0.29 \mu \mathrm{m}^{-1}$. The spatial Laplace transform $\Xi^{\text {mes }}(s)$ is shown by the complex $s$-plane surface plot of Fig. 1 . We then calculate a similar surface for varied values of $\left(\left|\tilde{\xi}^{1}\right|, \phi^{1}, k_{r}^{1}, k_{i}^{1}\right)$ and minimize the difference between the calculated and measured $s$-plane surface in Eq. (3) to end with $K^{1}=0.294+\mathrm{i} 3.71$ $\times 10^{-3} \mu \mathrm{m}^{-1}$, where the superscript denotes that this is the first identified mode (in this case only one mode is identified).

When $|\mathcal{M}| \neq 1$, each mode may, in practice, interact in the complex $s$-plane, which emphasizes the necessity of using a model involving all possible modes at a given frequency and the minimization of the previous cost function to efficiently determine all the possible $K^{m}$. This approach may offer advantages over related approaches in that highly attenuating and closely spaced modes may be distinguished from each other and from peaks associated with the finite measurement domain.

\section{APPLICATION TO GUIDED ELASTIC WAVES IN POROUS MATERIALS}

We first apply the SLaTCoW method to the case of guided elastic waves in porous materials. Porous materials are known to be highly dissipative due to viscothermal losses, interaction between the solid and fluid phases, and viscoelasticity of the skeleton. Seminal works by Boeckx et al. ${ }^{18}$ have paved the way for the characterization of the skeleton, porous material mechanical parameters by means of guided waves. These works mainly focus on the experimental recovery of the phase velocity $v_{\phi}^{m}=\omega / k_{r}^{m}$, in part, because of a lack of experimental data and analysis tools for the efficient determination of $k_{i}^{m}$. The present method is applied to experimentally determine both real and imaginary parts of the wavenumbers, thereby filling the existing gap in attenuation characterization capabilities, and enabling future works concerning the experimental measurement and modeling of viscoelastic parameters of porous materials.

The experimental setup, which is similar to the one used in Ref. 18, is depicted in Fig. 2(a). A high porosity $(\phi>0.95)$ melamine foam sample $85 \mathrm{~cm}$ long, $45 \mathrm{~cm}$ wide, and $5.5 \mathrm{~cm}$ thick is glued on a rigid backing. The excitation is provided by a shaker (Bruel and Kjaer type 4810), which is rigidly attached to the sample with a threaded steel rod ( $20 \mathrm{~mm}$ in length and $5 \mathrm{~mm}$ diameter) fixed to the shaker on one side and glued on a $1 \mathrm{~mm}$ thick aluminum plate of width $10 \mathrm{~cm}$ and height $1.5 \mathrm{~cm}$. This plate is cut at the edge opposite to the threaded steel rod and glued to the porous sample, creating a line source $15 \mathrm{~cm}$ from the edge of the sample. (a)
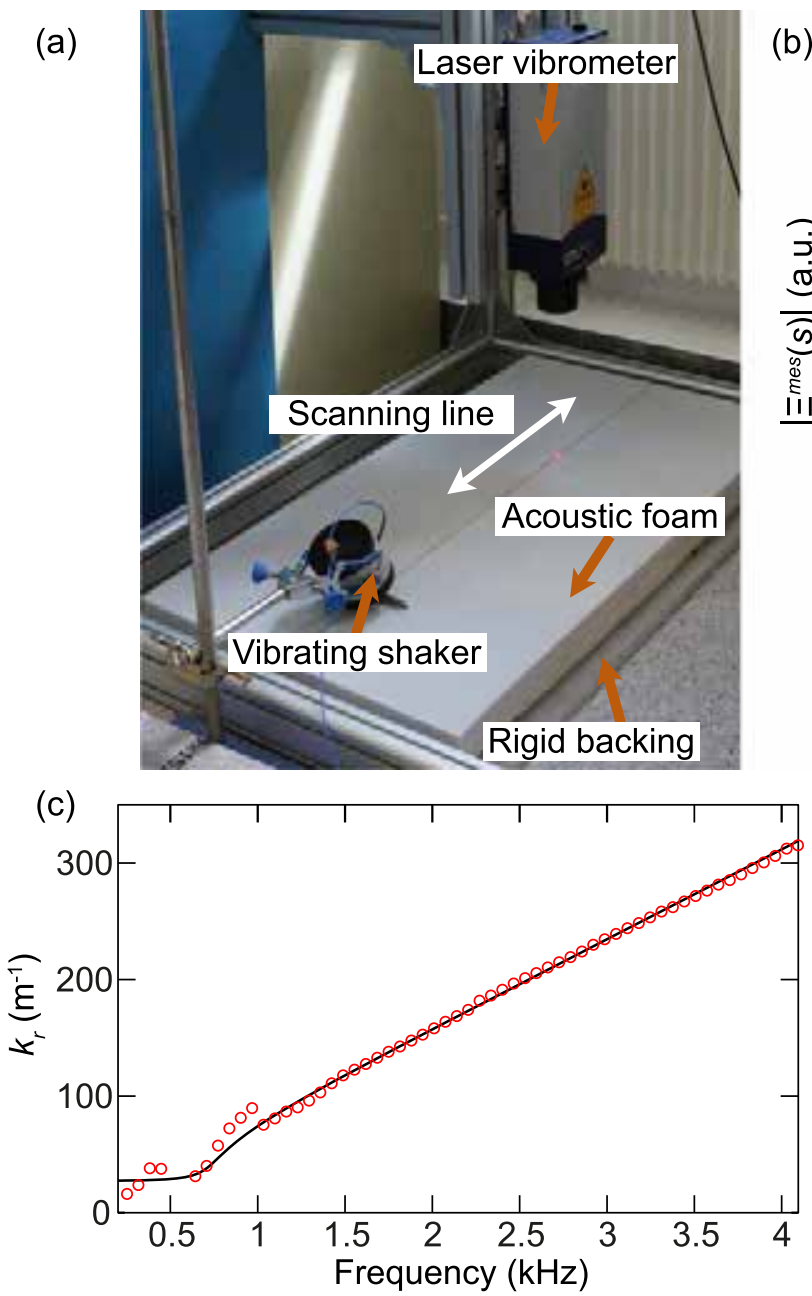

(b)
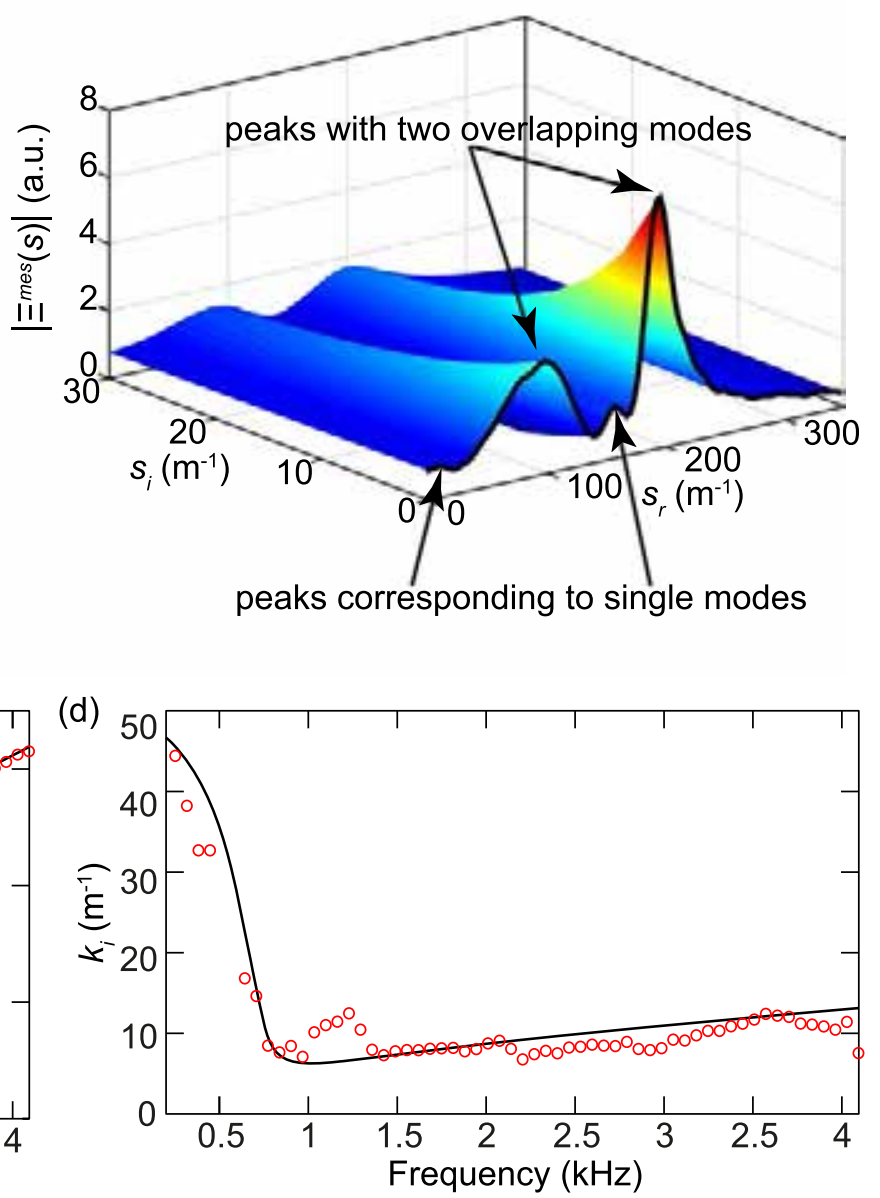

FIG. 2. (a) Photograph of the experimental setup. (b) Laplace transform $\Xi^{\text {mes }}(s)$ corresponding to $f=2453$ Hz. Arrows point out peaks, whose main component is a single mode, and peaks, whose main component are two modes overlapping. (c) $k_{r}$ and (d) attenuation $\left(k_{i}\right)$ of the first guided mode. Red circles depict the results obtained with the SLaTCoW method and solid lines depict the theoretical predictions. 
The resonance of this part was measured to be $4500 \mathrm{~Hz}$. While the measurement could be made at higher frequencies (up to $8000 \mathrm{~Hz}$ with a relatively good signal to noise ratio), the results are only shown for frequencies below this limit. The excitations are 300 sine functions equally spaced between $200 \mathrm{~Hz}$ and $4095 \mathrm{~Hz}$. The normal displacement $u_{n}(x)$ is acquired at 801 positions along a length of $L=$ $40 \mathrm{~cm}$ with a laser vibrometer (Polytec OFV-503) mounted on a one-dimensional moving stage, which moves the laser along the $x$-axis after each frequency measurement is accomplished. The vibrometer is connected to a spectral analyzer (Stanford Research Systems SR785), which allows us to directly measure $u_{n}(x)$ in the frequency domain. Each measurement is averaged 100 times.

The 1962 Biot model $^{26}$ parameters of the porous material, density $\rho$, porosity $\phi$, flow resistivity $\sigma$, viscous and thermal characteristic length $\Lambda$ and $\Lambda^{\prime}$ (respectively), real part of the shear modulus $N_{r}$, and Poisson ratio $\nu$ were determined independently using standard methods ${ }^{27}$ and are given in Table I. Viscous and thermal losses are accounted for by use of the Johnson-Champoux-Allard model, ${ }^{28,29}$ leading to complex and frequency dependent effective density and bulk modulus of the fluid phase. The imaginary part of the shear modulus $N_{i}$, which is usually considered constant in frequency, has been determined using the SLaTCoW method.

This type of measurement is known to be difficult due to large dissipation. More than 8 modes can be theoretically recovered over this frequency range, the velocities of which asymptotically tend towards the Rayleigh or shear velocities at high frequencies. Fig. 2(b) depicts the Laplace transform $\Xi^{\text {mes }}(s)$ at $f=2453 \mathrm{~Hz}$, where 6 modes can been seen. We choose to focus on the first guided mode, which was the one that was excited most efficiently. Extracting the complex wavenumber for this mode is typically difficult, as several modes are present in its vicinity. The procedure was applied to recover three modes around the first guided mode in order to remove the remaining components of the others. The real and the imaginary wavenumber, $k_{r}^{1}$ and $k_{i}^{1}$, respectively, versus frequency are found via the SLaTCoW method and plotted in Figs. 2(c) and 2(d), respectively. Theoretical predictions obtained using Stroh formalism $^{30,31}$ and a Müller algorithm agree well with the wavenumbers extracted using the SLaTCoW method when the complex shear modulus $N=N_{r}-\mathrm{i} N_{i}$ is fixed such that $N=38-\mathrm{i} 1.52 \mathrm{kPa}$ (damping factor $N_{i} / N_{r}=0.04$ ). This value is in accordance with the literature. ${ }^{32}$ This demonstrates the efficiency of the present method to discriminate modes when several are overlapping, and its effectiveness for extracting attenuation parameters. This paves the way for the extraction of new experimental information that enables the development of improved models of viscoelastic porous materials.

TABLE I. Material parameters for the melamine foam.

\begin{tabular}{lccccccc}
\hline \hline$\phi$ & $\rho\left(\mathrm{kg} \mathrm{m}^{-3}\right)$ & $\alpha_{\infty}$ & $\sigma\left(\mathrm{N} \mathrm{s} \mathrm{m}^{-4}\right)$ & $\Lambda(\mu \mathrm{m})$ & $\Lambda^{\prime}(\mu \mathrm{m})$ & $N(\mathrm{kPa})$ & $\nu$ \\
\hline 0.989 & 6.1 & 1 & 8060 & 215 & 215 & $38-\mathrm{i} 1.52$ & 0.3 \\
\hline \hline
\end{tabular}

\section{APPLICATION TO ZGV LAMB MODE IN A DURALUMIN PLATE}

We now apply the SLaTCoW method to obtain the complex dispersion curves of Lamb waves propagating in a $1.515 \mathrm{~mm}$ thick Duralumin plate. Particular attention is paid to the first symmetric $S_{1} S_{2} \mathrm{ZGV}$ Lamb mode. This mode is composed of two counter-propagating modes: the forward propagating $S_{1}$ Lamb mode and the backward propagating $S_{2 b}$ Lamb mode. ${ }^{33}$ In the ideal case of a non-absorbing material and free-standing plate, there are a unique wavenumber and frequency $\left(K^{S_{1} S_{2}}, f^{S_{1} S_{2}}\right)$ where these two modes coexist. At this point, the interference of $S_{1}$ and $S_{2 b}$ leads to the socalled $S_{1} S_{2}$-ZGV resonance. ${ }^{34}$ When the material absorption is significant or when there is leakage to the surrounding medium, the Lamb modes are inhomogeneous, their dispersion curves are no longer real valued, and their wavenumbers are complex. ${ }^{35-37}$ As previously noted for measurements made in a $50 \mu$ m-thick tungsten plate, attenuation leads to a separation of the $S_{1}$ and $S_{2 b}$ mode branches. ${ }^{38}$

Here, we illustrate a similar effect due to leakage. Using the SLaTCoW method, we estimate the complex dispersion branches in the vicinity of the ZGV point. The experimental setup is shown Fig. 3(a). A linear array ultrasound transducer probe (Imasonic SAS, $0.417 \mathrm{~mm}$ element pitch) made of 128 elements centered at $3.5 \mathrm{MHz}$, with a large frequency bandwidth, was used to generate Lamb waves in a Duralumin plate of thickness $1.515 \mathrm{~mm}$, width, and length $100 \mathrm{~mm}$. The probe was operated with the OPEN System of Lecoeur Electronique. The probe was coupled to the Duralumin plate using a thin layer of echographic gel approximately $0.150 \mathrm{~mm}$ thick. The first element of the array was used to generate an acoustic wave and then the 128 elements were used in receiver mode to record the normal displacement of the plate. The contact of the transducer array with the plate modifies the boundary conditions, induces leakage of the Lamb modes, and breaks the symmetry of the problem, such that the Lamb modes can no longer be separated in symmetric and antisymmetric families.

The results of the application of the SLaTCoW method to these experimental data concerning the two modes $\left(M^{3}\right.$ and $M^{4 b}$ ) within the ranges $k_{r} \in[0 ; 1.8] \mathrm{mm}^{-1}, k_{i} \in$ $[-0.5 ; 0.5] \mathrm{mm}^{-1}$, and $f \in[1.85 ; 2.03] \mathrm{MHz}$ are shown in Figs. 3(b) and 3(c). We denote the Lamb modes with a capital letter $M$, where the associated index indicates the mode number. The measured $M^{3}$ and $M^{4 b}$ Lamb modes have opposite phase velocities. While the real part of $K^{4 b}$ is negative, both wavenumbers $K^{3}$ and $K^{4 b}$ (where the superscripts of $K^{3}$ and $K^{4 b}$ correspond to modes $M^{3}$ and $M^{4 b}$ ) have positive imaginary parts as their amplitudes decay from the source to the receiver. However, for convenience, the backward mode wavenumber is represented with a positive real part and a negative imaginary part. Theoretical complex dispersion curves, using the classic Müller algorithm, are superimposed for comparison. Two cases have been considered: a freestanding Duralumin plate and a more realistic model. In both cases, the velocities of compressional and shear waves in Duralumin are set to $V_{L}=6380 \mathrm{~m} \mathrm{~s}^{-1}$ and $V_{T}=3080 \mathrm{~m} \mathrm{~s}^{-1}$, respectively, and the density is $\rho=2790 \mathrm{~kg} \mathrm{~m}^{-3}$. The 
(a)

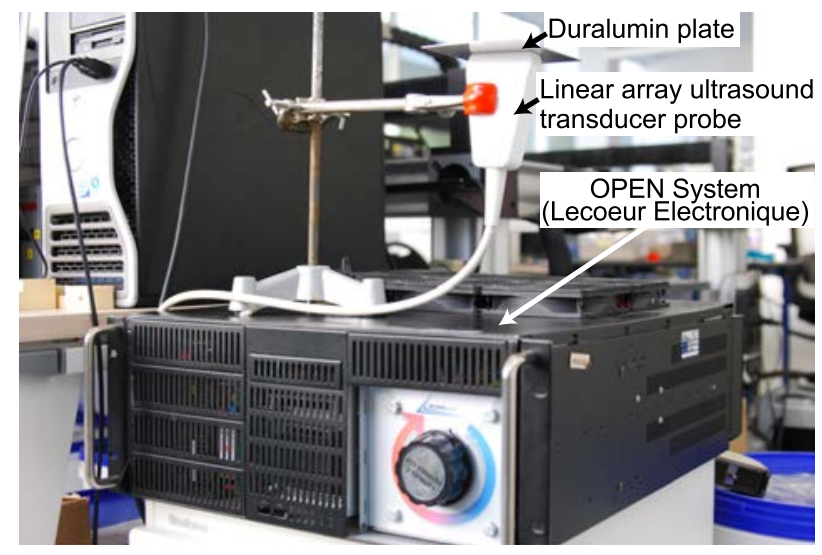

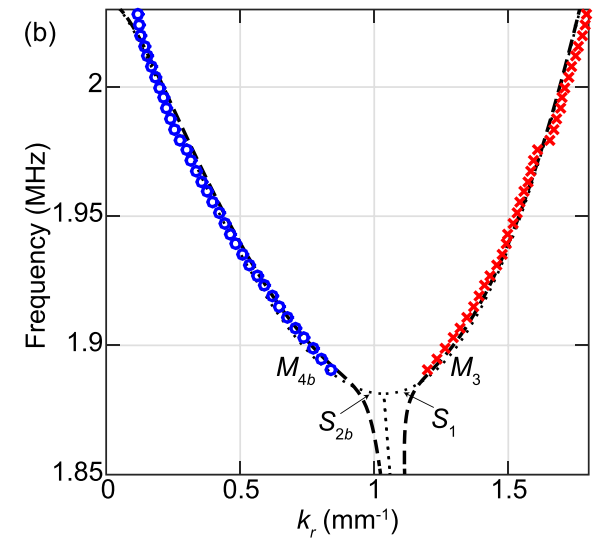

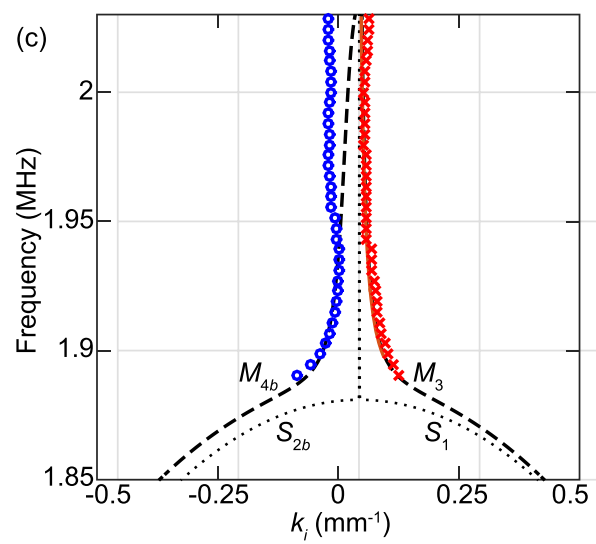

FIG. 3. (a) Photograph of the experimental setup. (b) and (c) Dispersion of Lamb waves in a $1.525 \mathrm{~mm}$ thick Duralumin plate: (b) real part and (c) imaginary part of the wavenumber. Markers denote the points identified using the SLaTCoW method. The chosen ranges, $k_{r} \in[0 ; 1.8] \mathrm{mm}^{-1}, k_{i} \in$ $[-0.5 ; 0.5] \mathrm{mm}^{-1}$, and $f \in[1.85 ; 2.03]$ $\mathrm{MHz}$, include two Lamb modes: $M^{3}$ (cross) and $M^{4 b}$ (circle). Theoretical complex dispersion curves using two different models are displayed for comparison: a model considering a free-standing Duralumin plate (dotted lines) and a more complex model (dashed lines). attenuation coefficient of Duralumin is taken from the literature as $0.9 \mathrm{~dB} \mathrm{~m}^{-1}$ at $5.86 \mathrm{MHz}^{15}$ Considering the dotted lines in Figs. 3(b) and 3(c), which represent the calculated dispersion curves accounting for intrinsic loss in a freestanding Duralumin plate, the differences between these curves and the SLaTCoW results are clear. This emphasizes the effects of the leakage of acoustic energy due to the contact of the transducer array, and the necessity to use a model that accounts for this. A more realistic model is composed of three perfectly bonded media: a layer of Duralumin in contact with a fluid layer (modeling the echographic gel), which is in contact with a half-space solid media representing the probe array. This model is used to calculate the theoretical dispersion curves shown as dashed lines in Figs. 3(b) and 3(c), using the previously stated properties for Duralumin. The $0.15 \mathrm{~mm}$ thick fluid layer is modeled as water, and it is assumed that no shear waves can propagate in it. The properties of the half-space have been chosen to be $V_{L}=3000 \mathrm{~m} \mathrm{~s}^{-1}, V_{T}=1300 \mathrm{~m} \mathrm{~s}^{-1}$, and $\rho=1100 \mathrm{~kg} \mathrm{~m}^{-3}$. No attenuation is assumed for both the fluid layer and the half-space. A good agreement between the theoretical curves and the points obtained using the SLaTCoW method can be seen. In Fig. 3(b), a separation of $M^{3}$ (cross) and $M^{4 b}$ (circle) branches is observed. These modes are typically not observed because of attenuation when approaching the position of the ZGV point. As shown in Fig. 3(b), as the position of the $\mathrm{ZGV}$ point of a free plate is approached, the imaginary part of the wavenumber and thus the attenuation become larger.

Although the chosen model may be considered overly simple, it is sufficient to reach good qualitative agreement with the results obtained using the SLaTCoW method. Note that the discrepancy in Fig. 3(c) between experiments and theory on the $M^{4 b}$ mode is due to the fact that this experimental branch is attenuated in the vicinity of $k_{r}=0$, making the SLaTCoW recovery harder to perform due to the very low signal-to-noise ratio. However, for the $M^{3}$ mode, where the signal-to-noise ratio is high, the agreement between the SLaTCoW results and the theoretical dispersion curves is excellent. The good qualitative agreement between the SLaTCoW results and the theoretical dispersion curves paves the way for new insights regarding the ZGV modes in freestanding plates and related multilayer systems.

\section{APPLICATION TO SURFACE ACOUSTIC WAVES IN A TWO-DIMENSIONAL MICROSCALE GRANULAR CRYSTAL ADHERED TO A SUBSTRATE}

We apply the SLaTCoW method to obtain the complex dispersion curves of SAWs propagating in a 2D microscale granular crystal adhered to a substrate. Ordered and reduceddimensional granular structures, often referred to as granular crystals, ${ }^{39-41}$ have proven to be systems of interest, as they represent an avenue for gaining a broader understanding of granular media dynamics, and have been suggested for use in stress wave manipulation and acoustic signal processing applications. ${ }^{40}$ While granular crystals have typically been constructed from macroscale particles, ${ }^{41}$ the acoustics of microscale granular crystals is an emerging field. ${ }^{24,42,43}$

In a recently published work, ${ }^{24}$ the resonant attenuation of SAWs propagating through a 2D microscale granular crystal adhered to a substrate was studied using a laser ultrasonic technique. A schematic of the sample and the laser ultrasonic setup used in the study of Ref. 24 is shown in Fig. 4. 


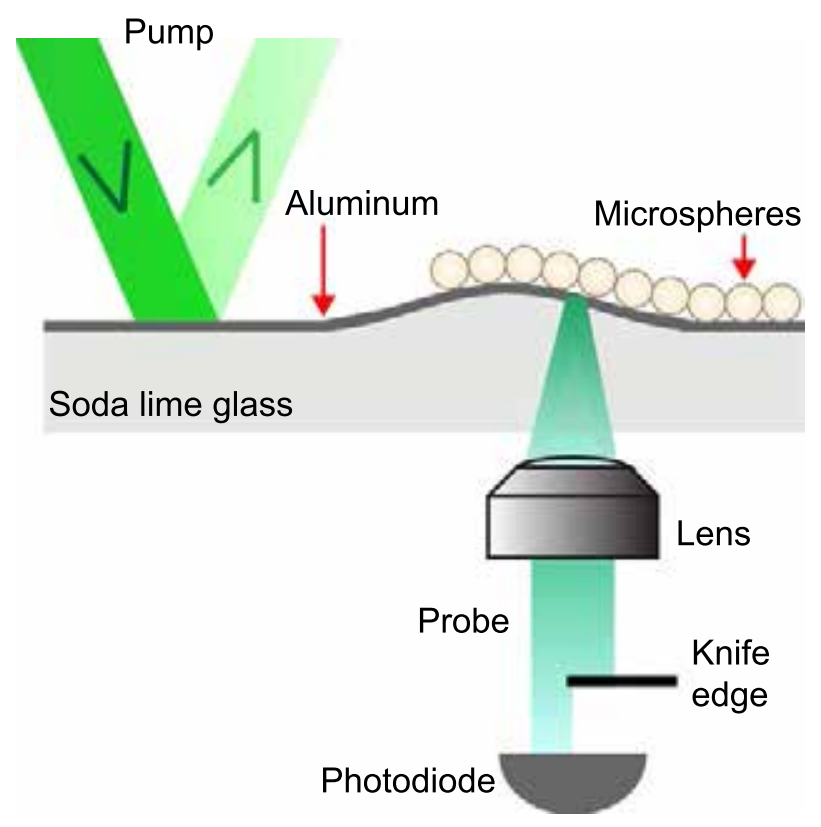

FIG. 4. Schematic of sample and laser ultrasonic experimental setup used in Ref. 24.

In summary, the microscale granular crystal is a monolayer of $2 \mu \mathrm{m}$ diameter silica spheres, which were assembled via a convective self-assembly process. ${ }^{44}$ An interface between regions with and without the monolayer was created using a microcontact printing technique. ${ }^{45}$ Surface acoustic waves were generated by focusing a pulsed laser into the sample surface (430 ps pulse duration, $532 \mathrm{~nm}$ optical wavelength, and $1.2 \mathrm{~mm} \times 20 \mu \mathrm{m}$ spot size), such that they traversed the interface, whereafter they were measured using a photodeflection technique (continuous wave laser, $514 \mathrm{~nm}$ optical wavelength, $6 \mu \mathrm{m}$ spot size) at 185 positions along a scan length of $740 \mu \mathrm{m}$. Additional experimental details can be found in Ref. 24. By calculating the 2D Fourier transform magnitudes of the spatiotemporal data corresponding to the
SAWs propagating in the region with the monolayer, the dispersion of SAWs propagating in the granular crystal was visualized. While this method did not separate the real and imaginary wavenumber components of the SAW dispersion, the calculated dispersion curves revealed the interaction between three contact-based resonances of the monolayer, ${ }^{24,46}$ including one with out-of-plane motion of the spheres and two with combined rotational and in-plane translational motion.

We apply the SLaTCoW method to the spatiotemporal measurement data obtained and presented in Ref. 24 (corresponding to the sample without any additional aluminum coating on top of the spheres). The resulting complex dispersion curves are shown in Fig. 5(a) $\left(k_{r}^{1}\right)$ and in Fig. 5(b) $\left(k_{i}^{1}\right)$. As can be seen in Fig. 5(a), an avoided crossing with the outof-plane contact resonance $\left(f_{N}\right)$ of the monolayer is present and is the only visible feature disturbing the straight line corresponding to Rayleigh waves propagating in the substrate. We compare the dispersion curves calculated from experimentally obtained data with those calculated in Ref. 24 using a lossless theoretical model, ${ }^{46}$ and find a reasonable agreement in the neighborhood of the out-of-plane contact resonance. As in Ref. 24, avoided crossings due to the two rotational-translational modes (including the rotation dominated resonance $f_{R H}$ and the translation dominated resonance $\left.f_{H R}\right)$ are both absent. In contrast, the two rotationaltranslational resonances are both clearly visible in the dispersion curves corresponding to the imaginary part of the wavenumber shown in Fig. 5(b) as smaller peaks surrounding the large out-of-plane resonance.

This work and the SLaTCoW method pave the way for the development of new models of microscale granular dynamics, which may find use in discerning the contributions of various sources of loss observed in experiments. ${ }^{24,42}$ For example, there is currently no model for the imaginary part of $K^{m}$ for the 2D microscale granular crystal adhered to a
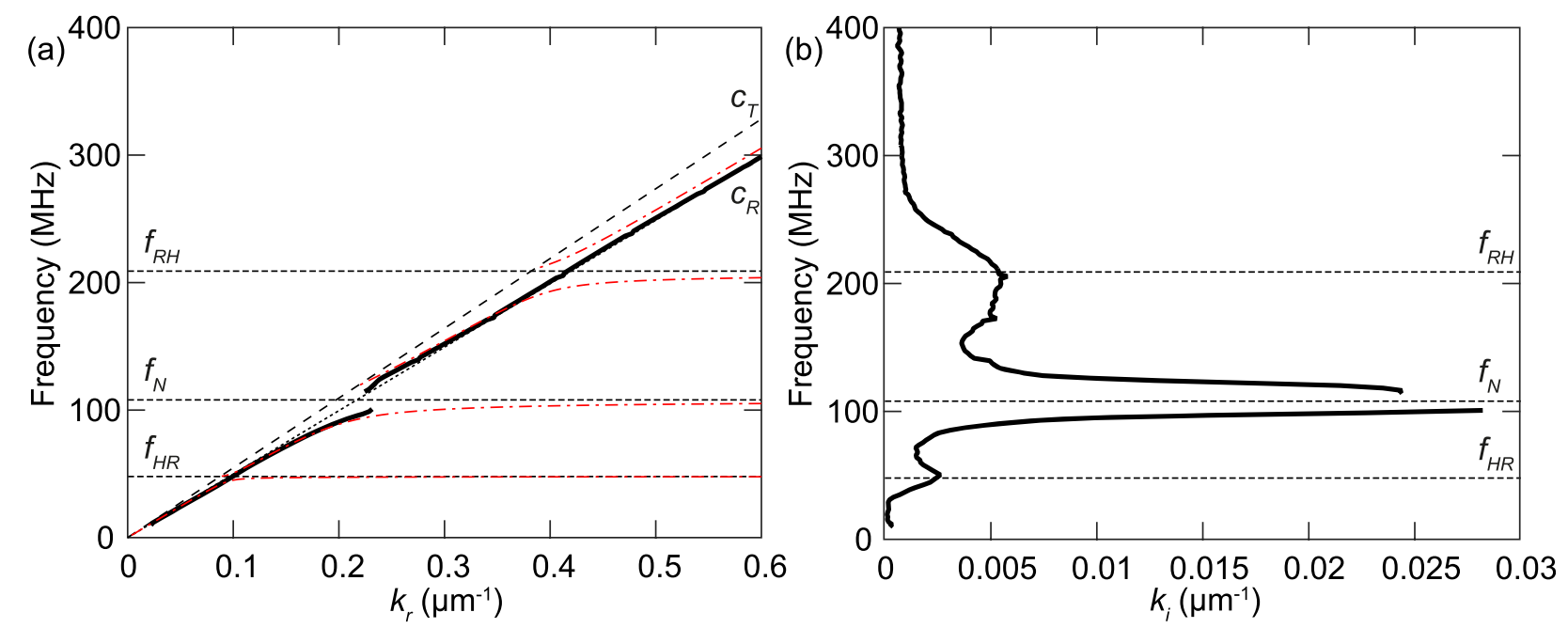

FIG. 5. (a) Dispersion curves of SAWs propagating in a substrate with an adhered microsphere monolayer, corresponding to the real part of the wavenumber, $k_{r}^{1}$. Solid lines are dispersion curves calculated using the SLaTCoW method. Red dash-dotted lines are the dispersion curves calculated using a lossless model and the fitted contact resonance frequencies (denoted by the horizontal dotted lines). Rayleigh $\left(c_{R}\right)$ and transverse $\left(c_{T}\right)$ wave speeds are the straight dotted and dashed lines, respectively. (b) Dispersion curves of SAWs propagating in a substrate with an adhered microsphere monolayer calculated using the SLaTCoW method, corresponding to the imaginary part of the wavenumber, $k_{i}^{1}$. Horizontal dotted lines denote the fitted contact resonance frequencies. Solid lines in both panels are computed using experimental data originally obtained and presented in Ref. 24. 
substrate. This demonstrates the efficacy of the method to advance relevant modeling efforts.

\section{CONCLUSION}

A method for the recovery of complex spatial frequency domain information from spatiotemporal data is presented. This method, named SLaTCoW (Spatial Laplace Transform for COmplex Wavenumber recovery), is based on a spatial Laplace transform of the measured wave field in the frequency domain, instead of the usual spatial Fourier transform. The Laplace transform, providing information on both the real and imaginary parts of the poles, is analyzed by the minimization of a correctly chosen cost function. This allows the reconstruction of complex wavenumbers (as well as the complex amplitude) of the modes, even when they are interacting with other modes. The SLaTCoW method was applied to three completely different dispersive and attenuating systems, involving three different set-ups in three different frequency ranges, and showed use in the analysis of attenuation phenomena occurring in each system. This method provides information that cannot be obtained from 2D Fourier transforms, including the separation of modes that are almost overlapping, as well as amplitude independent information. The SLaTCoW method paves the way for new theoretical developments in various fields of physical acoustics such as attenuating and locally resonant materials.

\section{ACKNOWLEDGMENTS}

J.-P. Groby gratefully acknowledges the support from the ANR Project METAUDIBLE No. ANR-13-BS09-0003 co-founded by ANR and FRAE. J.-P. Groby and A. Duclos are grateful for the support of the LMAc projects DECIMAP and PAVNat. N. Boechler, A. Khanolkar, S.P. Wallen, and M. Abi Ghanem acknowledge the support by the U.S. National Science Foundation (Grant No. CMMI-1333858), the U.S. Army Research Office (Grant No. W911NF-15-10030), and the University of Washington Royalty Research Foundation. M. Hiraiwa acknowledges the support from the National Science Foundation Graduate Research Fellowship Program under Grant No. DGE-1256082. The contribution by J. Laurent and C. Prada was supported by LABEX WIFI (Laboratory of Excellence ANR-10-LABX-24) within the French Program Investments for the Future under Reference No. ANR-10-IDEX-0001-02 PSL*.

${ }^{1}$ R. D. S. Yadava, R. Kshetrimayum, and M. Khaneja, "Multifrequency characterization of viscoelastic polymers and vapor sensing based on saw oscillators," Ultrasonics 49, 638-645 (2009).

${ }^{2}$ C. Ruppert, J. Neumann, J. B. Kinzel, H. J. Krenner, A. Wixforth, and M. Betz, "Surface acoustic wave mediated coupling of free-space radiation into surface plasmon polaritons on plain metal films," Phys. Rev. B 82, 081416 (2010).

${ }^{3}$ H.-X. Sun, S.-Y. Zhang, and B.-Q. Xu, "Influence of viscoelastic property on laser-generated surface acoustic waves in coating-substrate systems," J. Appl. Phys. 109, 073107 (2011).

${ }^{4}$ J. H. Page, P. Sheng, H. P. Schriemer, I. Jones, X. Jing, and D. A. Weitz, "Group velocity in strongly scattering media," Science 271, 634-637 (1996).

${ }^{5}$ Z. Liu, C. T. Chan, P. Sheng, A. L. Goertzen, and J. H. Page, "Elastic wave scattering by periodic structures of spherical objects: Theory and experiment," Phys. Rev. B 62, 2446-2457 (2000).
${ }^{6}$ H. Pichard, A. Duclos, J.-P. Groby, V. Tournat, and V. E. Gusev, "Twodimensional discrete granular phononic crystal for shear wave control," Phys. Rev. B 86, 134307 (2012).

${ }^{7}$ R. J. P. Engelen, D. Mori, T. Baba, and L. Kuipers, "Subwavelength structure of the evanescent field of an optical bloch wave," Phys. Rev. Lett. 102, 023902 (2009).

${ }^{8}$ V. Romero-García, J. V. Sánchez-Pérez, S. Castiñeira-Ibáñez, and L. M. Garcia-Raffi, "Evidences of evanescent bloch waves in phononic crystals," Appl. Phys. Lett. 96, 124102 (2010).

${ }^{9}$ R. Schmidt, "Multiple emitter location and signal parameter estimation," IEEE Trans. Antenna Propag. 34, 276-280 (1986).

${ }^{10}$ E. C. Shang, H. P. Wang, and Z. Y. Huang, "Waveguide characterization and source localization in shallow water waveguides using the prony method,” J. Acoust. Soc. Am. 83, 103-108 (1988).

${ }^{11}$ R. Roy, A. Paulraj, and T. Kailath, "Esprit-a subspace rotation approach to estimation of parameters of cisoids in noise," IEEE Trans. Acoust. Speech 34, 1340-1342 (1986).

${ }^{12}$ R. Roy and T. Kailath, "Esprit-estimation of signal parameters via rotational invariance techniques," IEEE Trans. Acoust. Speech 37, 984-995 (1989).

${ }^{13}$ F. Schöpfer, F. Binder, A. Wöstehoff, T. Schuster, S. Ende, S. Föll, and R. Lammering, "Accurate determination of dispersion curves of guided waves in plates by applying the matrix pencil method to laser vibrometer measurement data," CEAS Aeronaut. J. 4, 61-68 (2013).

${ }^{14}$ F. Philippe, P. Roux, and D. Cassereau, "Iterative high-resolution wavenumber inversion applied to broadband acoustic data," IEEE Trans. Ultrason. Ferroelectr. Freq. Control 55, 2306-2311 (2008).

${ }^{15}$ C. Prada, D. Clorennec, and D. Royer, "Power law decay of zero group velocity lamb modes," Wave Motion 45, 723-728 (2008).

${ }^{16}$ G. Rix, C. Lai, and A. Spang, Jr., "In situ measurement of damping ratio using surface waves," J. Geotech. Geoenviron. Eng. 126, 472-480 (2000).

${ }^{17}$ J. F. Allard, O. Dazel, J. Descheemaeker, N. Geebelen, L. Boeckx, and W. Lauriks, "Rayleigh waves in air saturated axisymmetrical soft porous media,” J. Appl. Phys. 106, 014906 (2009).

${ }^{18}$ L. Boeckx, P. Leclaire, P. Khurana, C. Glorieux, W. Lauriks, and J. F. Allard, "Guided elastic waves in porous materials saturated by air under lamb conditions," J. Appl. Phys. 97, 094911 (2005).

${ }^{19}$ K. J. Ellefsen, C. H. Cheng, and K. M. Tubman, "Estimating phase velocity and attenuation of guided waves in acoustic logging data," Geophysics 54, 1054-1059 (1989).

${ }^{20} \mathrm{~S}$. Foti, "Small-strain stiffness and damping ratio of pisa clay from surface wave tests," Géotechnique 53, 455-461 (2003).

${ }^{21}$ A. S. Misbah and C. L. Strobbia, "Joint estimation of modal attenuation and velocity from multichannel surface wave data," Geophysics 79, EN25-EN38 (2014).

${ }^{22}$ J.-G. Minonzio, J. Foiret, M. Talmant, and P. Laugier, "Impact of attenuation on guided mode wavenumber measurement in axial transmission on bone mimicking plates," J. Acoust. Soc. Am. 130, 3574-3582 (2011).

${ }^{23}$ G. Rix, C. Lai, and S. Foti, "Simultaneous measurement of surface wave dispersion and attenuation curves," Geotech. Test. J. 24, 350-358 (2001).

${ }^{24}$ M. Hiraiwa, M. Abi Ghanem, S. P. Wallen, A. Khanolkar, A. A. Maznev, and N. Boechler, "Complex contact-based dynamics of microsphere monolayers revealed by resonant attenuation of surface acoustic waves," Phys. Rev. Lett. 116, 198001 (2016).

${ }^{25}$ J. Prisutova, K. Horoshenkov, J.-P. Groby, and B. Brouard, "A method to determine the acoustic reflection and absorption coefficients of porous media by using modal dispersion in a waveguide," J. Acoust. Soc. Am. 136, 2947-2958 (2014).

${ }^{26} \mathrm{M}$. Biot, "Mechanics of deformation and acoustic propagation in porous media," J. Appl. Phys. 33, 1482-1498 (1962).

${ }^{27}$ J. F. Allard and N. Atalla, Propagation of Sound in Porous Media: Modelling Sound Absorbing Materials 2e (Wiley, Chichester, 2009), Chap. 5.

${ }^{28}$ D. L. Johnson, J. Koplik, and R. Dashen, "Theory of dynamic permeability and tortuosity in fluid-saturated porous media," J. Fluid Mech. 176, 379-402 (1987).

${ }^{29}$ Y. Champoux and J. Allard, "Dynamic tortuosity and bulk modulus in airsaturated porous media,” J. Appl. Phys. 70, 1975-1979 (1991).

${ }^{30}$ G. Gautier, L. Kelders, J. P. Groby, O. Dazel, L. De Ryck, and P. Leclaire, "Propagation of acoustic waves in a one-dimensional macroscopically inhomogeneous poroelastic material," J. Acoust. Soc. Am. 130, 1390-1398 (2011). 
${ }^{31}$ O. Dazel, J.-P. Groby, B. Brouard, and C. Potel, "A stable method to model the acoustic response of multilayered structures," J. Appl. Phys. 113, 083506 (2013).

${ }^{32}$ L. Jaouen, A. Renault, and M. Deverge, "Elastic and damping characterizations of acoustical porous materials: Available experimental methods and applications to a melamine foam," Appl. Acoust. 69, 1129-1140 (2008).

${ }^{33}$ D. Clorennec, C. Prada, D. Royer, and T. W. Murray, "Laser impulse generation and interferometer detection of zero group velocity lamb mode resonance," Appl. Phys. Lett. 89, 024101 (2006).

${ }^{34}$ C. Prada, D. Clorennec, and D. Royer, "Local vibration of an elastic plate and zero-group velocity lamb modes," J. Acoust. Soc. Am. 124, 203-212 (2008).

${ }^{35}$ R. D. Mindlin and M. A. Medick, "Extensional vibrations of elastic plates," J. Appl. Mech. 26, 561-569 (1959).

${ }^{36}$ R. D. Mindlin and J. Yang, "Solutions of the three-dimensional equations," in An Introduction to the Mathematical Theory of Vibrations of Elastic Plates (World Scientific, 2012), Chap. 2, pp. 23-78.

${ }^{37}$ B. Auld, Acoustic Fields and Waves in Solids (R. E. Krieger Publishing Company, Malabar, FL, 1990), Chap. 10, pp. 73-94.

${ }^{38}$ C. Prada, O. Balogun, and T. W. Murray, "Laser-based ultrasonic generation and detection of zero-group velocity lamb waves in thin plates," Appl. Phys. Lett. 87, 194109 (2005).
${ }^{39} \mathrm{~V}$. Nesterenko, Dynamics of Heterogeneous Materials (Springer-Verlag New York, 2001).

${ }^{40} \mathrm{G}$. Theocharis, N. Boechler, and C. Daraio, "Nonlinear periodic phononic structures and granular crystals," in Acoustic Metamaterials and Phononic Crystals (Springer, Berlin, 2013), pp. 217-251.

${ }^{41}$ M. Porter, P. Kevrekidis, and C. Daraio, "Granular crystals: Nonlinear dynamics meets materials engineering," Phys. Today 68(11), 44-50 (2015).

${ }^{42}$ N. Boechler, J. K. Eliason, A. Kumar, A. A. Maznev, K. A. Nelson, and N. Fang, "Interaction of a contact resonance of microspheres with surface acoustic waves," Phys. Rev. Lett. 111, 036103 (2013).

${ }^{43}$ P. Ruello, A. Ayouch, G. Vaudel, T. Pezeril, N. Delorme, S. Sato, K. Kimura, and V. E. Gusev, "Ultrafast acousto-plasmonics in gold nanoparticle superlattices," Phys. Rev. B 92, 174304 (2015).

${ }^{44}$ J. Sun, C.-J. Tang, P. Zhan, Z.-I. Han, Z.-S. Cao, and Z.-L. Wang, "Fabrication of centimeter-sized single-domain two-dimensional colloidal crystals in a wedge-shaped cell under capillary forces," Langmuir $\mathbf{2 6}$, 7859-7864 (2010).

${ }^{45}$ B. H. Lee, H. Shin, and M. M. Sung, "Patterning a two-dimensional colloidal crystal by water-mediated particle transfer printing," Chem. Mater. 19, 5553-5556 (2007).

${ }^{46}$ S. P. Wallen, A. A. Maznev, and N. Boechler, "Dynamics of a monolayer of microspheres on an elastic substrate,” Phys. Rev. B 92, 174303 (2015). 